\title{
Diferenças de Gênero na Preferência de Pares e Brincadeiras de Crianças
}

\author{
Gender Differences in Children's Peer and Play Preferences
}

\author{
Aline Beckmann de Castro Menezes \& Regina Célia Sousa Brito \\ Universidade Federal do Pará, Belém, Brasil
}

\begin{abstract}
Resumo
O dimorfismo sexual na preferência de pares e brincadeiras infantis é registrado desde idades precoces. Crianças com padrões atípicos do seu sexo tendem a apresentar dificuldades de socialização. Investigaramse tais preferências utilizando dois instrumentos padronizados: Entrevista Estruturada de Preferências de Parcerias e Estilos de Brincadeiras e Desenho da Figura Humana. Participaram 234 crianças, de 9 a 11 anos, sendo 120 do sexo feminino. Observou-se correlação elevada entre o sexo da criança e o sexo do par preferido, bem como com brincadeiras típicas do seu sexo e o sexo da pessoa desenhada corroborando dados similares obtidos pela literatura internacional. Conclui-se que crianças entre 9 e 11 anos apresentam predominância de padrões típicos de gênero sem que os mesmos sejam rígidos.

Palavras-chave: Dimorfismo sexual, mensuração do comportamento, diferenças de gênero, brincadeira.

Abstract

Sexual dimorphism in peer and play preference has been observed in very early ages. Children with atypical gender behavior usually present socialization difficulties. Their preferences were investigated through two standardized instruments: Playmate and Play Style Preferences Structured Interview and Human Figure Drawing. The participants were 234 children, from 9 to 11 years old, 120 girls. It was observed high correlation between child sex and peer sex as well as type of play and sex of the person drawn, corroborating similar data from international literature. It has been concluded that children between 9 and 11 years old have gender typical choice patterns, but they are not inflexible.

Keywords: Sexual dimorphism, measurement of behavior, gender differences, child's play.
\end{abstract}

Padrões sexualmente dimórficos são aqueles comportamentos que tendem a se manifestar de forma diferenciada de acordo com o sexo do indivíduo em questão. $\mathrm{O}$ estudo destes padrões na infância tem privilegiado o contexto da brincadeira (Burghardt, 2004), seja diretamente - através da observação (ver, por exemplo, Bianchi \& Bakeman, 1978) - ou indiretamente - através de entrevistas e instrumentos padronizados (ver, por exemplo, Finnegan, Niccols, Zacher, \& Hood, 1991). Esta ênfase deve-se ao fato de que a brincadeira é atividade central na vida infantil, possibilitando a construção de sua cultura e identidade a partir da interação com os pares (Conti \& Sperb, 2001).

Já em 1977, Fagot identificou padrões sexualmente dimórficos nas preferências infantis (entre três e quatro anos). Meninas tenderam a preferir brincar de arte, cozinha, boneca e de vestir fantasias, enquanto meninos tenderam a preferir brincar com blocos de construção, brinquedos transportadores e brincadeiras externas na

"Endereço para correspondência: Faculdades Integradas Ipiranga, Núcleo de Apoio Psicopedagógico, Av. Almte Barroso, 777, Marco, Belém, PA, Brasil 66000-000. E-mail: alinebcm@gmail.com e rcsb@uol.com.br areia. Segundo este autor, de um modo geral, comportamentos atípicos do sexo tendiam a ser punidos por professores e pares quando emitidos por meninos, mas não quando por meninas. Para ambos os sexos, tais comportamentos raramente recebiam encorajamento sendo, usualmente, ignorados.

Liss (1981) investigou tanto a preferência por tipo de brinquedo quanto a forma de brincar. Para tal, expôs crianças de sexos diferentes aos mesmos brinquedos. Meninos tendiam a preferir brinquedos tipicamente masculinos ou neutros, enquanto meninas tendiam a preferir brinquedos femininos. Quando meninas brincavam com brinquedos masculinos, o estilo de brincadeira tendia a ser similar ao de meninos. Contudo, meninos brincando com brinquedos femininos, tendiam a adotar um estilo de brincadeira diferente, favorecendo habilidades espaciais, por exemplo. Com isso, a autora discute que ao escolher um parceiro para brincar, a criança pode não utilizar o sexo da outra criança em si como critério, mas o estilo de brincadeira que a outra criança possui. O que, inclusive, é congruente com a maior inserção de meninas nos grupos masculinos do que o inverso (Menezes et al., 2010).

O mesmo padrão de escolha de brinquedos foi encontrado por Jacklin, Di Pietro e Maccoby (1984) em crian- 
ças de aproximadamente 3,5 anos, sendo a preferência fortalecida pelo comportamento parental, que tende a iniciar brincadeiras congruentes com o sexo da criança.

Mais recentemente, estudos encontraram evidências de precocidade do dimorfismo. No estudo desenvolvido por Alexander, Wilcox e Woods (2009), crianças entre quatro e oito meses de idade apresentaram padrões sexualmente dimórficos de atenção a objetos (caminhão e boneca). Apesar de nem todos os resultados obtidos terem sido estatisticamente significativos, este dado indica que o sexo do bebê afeta a atração por diferentes aspectos dos objetos.

Bailey, Bechtold e Berenbaum (2002), estudaram meninas masculinizadas $\left(\right.$ tomboy $\left.^{1}\right)$. As participantes desse estudo apresentavam as principais diferenças em três aspectos: preferência pelo par na brincadeira; interesses e atividades sexualmente dimórficas; e identidade de gênero. $\mathrm{O}$ estudo comparou respostas e padrões de meninas masculinizadas, com os de suas irmãs femininas e seus irmãos. Foram obtidas correlações significativas, indicando que, ao contrário de seus irmãos, as meninas masculinizadas tendiam a preferir se relacionar com pessoas do sexo oposto, com quem compartilhavam interesses e identificação.

Em um estudo realizado com crianças gêmeas holandesas entre 7 e 10 anos de idade, Beijsterveldt, Hudziak e Boomsma (2006) observaram que a não conformidade de gênero é mais comum do que o Transtorno de Identidade de Gênero (GID), o qual implica não só na identificação com o sexo oposto mas em sensações de desconforto e inadequação com o próprio sexo (American Psychiatric Association, 1995). Isto é, comportamentos atípicos do sexo não significam necessariamente a presença de GID, podendo o indivíduo sentir-se à vontade com o próprio sexo, mesmo comportando-se diferentemente da média de seus pares.

$\mathrm{Na}$ escolha de pares de brincadeiras, as crianças tendem não só a preferir pares do mesmo sexo, como a evitar aqueles do sexo oposto (Golombok et al., 2008). A estereotipia comportamental de acordo com o sexo foi medida longitudinalmente por este autor, mantendo-se estável e ainda se intensificando, em participantes tanto com comportamentos sexualmente típicos quanto atípicos. Os autores interpretam os resultados como evidência de influências biológicas sobre as preferências precocemente identificadas (aos 2,5 anos de idade) e do seu fortalecimento a partir da interação com pares e da influência cultural.

Defendendo a atuação da socialização ou do desenvolvimento cognitivo sobre as preferências, Jadva, Hines e Golombok (2010) identificaram que crianças entre um e

\footnotetext{
${ }^{1}$ Tomboy é uma expressão em inglês, sem equivalente direto em português, que significa pessoa do sexo feminino que apresenta um conjunto amplo e variado de características típicas do sexo masculino.
}

dois anos tendem a preferir brincadeiras sexualmente dimórficas (meninos preferindo carros e meninas preferindo bonecas).

Pode-se perceber, por esta revisão, que há evidências consolidadas na literatura sobre o dimorfismo sexual na preferência de pares e brincadeiras infantis, desde idades precoces (aos quatro meses, como em Alexander et al., 2009). Há três correntes principais que buscam a explicação destas diferenças: aprendizagem social; desenvolvimento cognitivo; aspectos biológicos e evolucionistas.

A proposta dos teóricos da aprendizagem social, como Bussey e Bandura (1999), é de que a criança já nasce em um mundo sexualmente dividido e é estimulada diferencialmente, sendo reforçados padrões comportamentais típicos do sexo e punidos ou ignorados os comportamento atípicos. Desta forma, as diferenças de gênero seriam socialmente construídas a partir da interação com os outros, especialmente adultos (Jadva et al., 2010).

A perspectiva cognitiva parte do pressuposto de que a criança forma categorias mentais de feminino e masculino, de modo que ao se identificar como membro de uma destas categorias, tende a procurar atividades e pares compatíveis com sua própria identificação (Jadva et al., 2010). O maior desafio desta perspectiva é explicar como e a partir de que idades tais constructos (masculinidade e feminilidade) se desenvolveriam.

A perspectiva biológica predominante refere-se à ação de hormônios pré-natais que influenciariam a suscetibilidade individual a determinadas estimulações. Assim, a partir desta ação, crianças do sexo masculino teriam, por exemplo, maior agressividade e maior habilidade espacial, enquanto as do sexo feminino processariam melhor a imagem de rostos e teriam melhor coordenação motora fina (Alexander, 2003).

Alexander (2003) discute a perspectiva evolucionista de compreensão do padrão sexualmente dimórfico de preferência de brincadeiras infantis. Segundo esta autora, tais preferências são manifestações precoces do papel de gênero a ser desenvolvido na vida adulta. No Ambiente de Adaptação Evolutiva (AAE) as pressões exercidas sobre machos e fêmeas eram diferentes entre si, provocando a seleção de diferentes mecanismos, relacionados a padrões comportamentais distintos observados até hoje na espécie humana. Por exemplo, parcerias masculinas eram importantes para momentos como caça e proteção de recursos (requerendo habilidades espaciais), enquanto parcerias femininas relacionavam-se à coleta de alimentos e ao cuidado parental (requerendo habilidades ligadas à identificação de formas e cores e memória da localização de objetos, favorecendo o forrageio). Tais diferenças implicariam em crianças do sexo masculino terem preferência por brinquedos como bolas e carros (relacionados ao movimento) e crianças do sexo feminino prefeririam cores quentes e bonecas (ligado ao forrageio e ao cuidado parental). 
Evidências que corroboram esta perspectiva podem ser obtidas através de pesquisas que identificaram o dimorfismo sexual precoce, indicando que a criança ainda não possui uma concepção sobre a sua classificação sexual, contudo já apresenta padrões de preferência distintos. Assim, a ação de hormônios pré-natais pode afetar o desenvolvimento do sistema nervoso central, de modo que haveria um padrão sexualmente dimórfico, selecionado ao longo da evolução da espécie, para a suscetibilidade a formas e estimulações específicas.

Concordando com esta perspectiva, Van de Beek, van Goozen, Buitelaar e Cohen-Kettenis (2009) apresentam dados de dimorfismo na preferência por brinquedos aos 13 meses de idade e afirmam que esta precocidade decorre de alterações no desenvolvimento cerebral fetal a partir da influência de hormônios pré-natais (índices significativamente superiores de testosterona no líquido amniótico de fetos masculinos e de estradiol no de fetos femininos).

Shirley e Campbell (2000) encontraram diferenças nas preferências infantis aos três meses de idade. Afirmam que "a criança traz tanto ao ambiente quanto o ambiente traz à criança" (p. 17), isto é, a criança tanto aprende as diferenças de gênero a partir de um ambiente assim dividido e estruturado quanto traz para esse ambiente já suas predisposições e predileções. Desta forma, compreender a existência de características biologicamente definidas não significa que o meio não exerça um importante papel reforçando estas diferenças. Processos sociais e cognitivos se estabeleceriam sobre uma base de preferências pré-existentes por categorias de brinquedos e brincadeiras (Alexander et al., 2009).

Uma evidência do papel do ambiente é encontrada no estudo de Bianchi e Bakeman (1978). Estes autores relatam que evidências dos padrões sexualmente dimórficos remontam à década de 1930, sendo reiteradamente estudados e corroborados por diferentes pesquisadores, com múltiplas metodologias e em diversas culturas, mas que encontraram uma significativa diminuição da estereotipia sexual em padrões de brincadeira quando a escola era planejada de forma a não enfatizar as diferenças sexuais, mas a estimular a interação intersexual.

Esta visão interacionista foi fortalecida 30 anos depois por Golombok et al. (2008), ao identificar que a estereotipia dos padrões sexualmente dimórficos tende a crescer com a interação restrita a indivíduos do mesmo sexo. De acordo com Iervolino, Hines, Golombok, Rust e Plomin (2005), hoje há um consenso de que a socialização, o desenvolvimento cognitivo e fatores biológicos contribuem interativamente com o desenvolvimento do comportamento sexualmente dimórfico. Investigando mais de 8 mil indivíduos, estes autores encontraram que há contribuições tanto genéticas quanto ambientais, sendo as primeiras mais fortes sobre características pessoais e as segundas sobre tipos de atividades e interesses.
Outro estudo que identifica o papel ambiental no fortalecimento do dimorfismo sexual é o de Pomerleau, Bolduc, Malcuit e Cossete (1990). Estes autores observaram que pais planejam o ambiente para recém-nascidos de acordo com o sexo da criança, expondo-os desde os 5 meses de idade a diferentes cores, objetos, sons, etc.

Pode-se perceber que com diferentes perspectivas teóricas e usando metodologias diversas, os dados existentes na literatura da área apontam para a existência de padrões comportamentais sexualmente dimórficos, cuja origem pode ser explicada tanto por fatores biológicos, que remetem ao passado evolutivo da espécie, quanto por fatores psicossociais, referentes às interações com indivíduos do mesmo sexo e do sexo oposto e em contato com as normas culturais. Para verificar a universalidade deste dimorfismo, contudo, é importante que sejam feitas replicações transculturais dos estudos citados, comparando os resultados obtidos.

O estudo das diferenças de gênero na infância é de grande relevância por diversos motivos. A maior parte da vida social é estruturada a partir da divisão de gênero, com expectativas sociais referentes a padrões comportamentais tipicamente masculinos ou femininos (Bussey \& Bandura, 1999).

Além disso, crianças com padrões atípicos do seu sexo tendem a apresentar dificuldades de socialização e integração com seus pares. Por exemplo, Fridell, OwenAnderson, Johnson, Bradley e Zucker (2006) observaram que meninos identificados como não conformes de gênero possuíam maior dificuldade em se inserir socialmente do que os demais meninos, limitando as interações desenvolvidas com outras crianças, principalmente as do sexo masculino.

O impacto da não conformidade de gênero foi estudado por Cohen-Kettenis, Owen, Kaijseer, Bradley e Zucker (2003). Observaram que crianças diagnosticadas com GID apresentaram maior número de problemas comportamentais gerais e menor competência social, com elevadas alterações emocionais e déficits comportamentais O ostracismo social (no sentido de isolamento generalizado por pares) foi correlacionado com maior número de problemas comportamentais, especialmente entre crianças do sexo masculino devido às relações sociais mais empobrecidas que as do sexo feminino (o que reflete a rejeição social de meninos femininos já mencionada anteriormente). Resumidamente, os autores concluíram que tanto para as crianças do sexo masculino quanto as do feminino, os problemas referentes às relações com os pares foram o mais forte preditor de problemas comportamentais

Van Beek, van Dolderen e Dubas (2006) identificaram uma relação entre problemas nas relações sociais e a origem da depressão. Para estes autores, a falta de habilidades sociais pode levar à rejeição pelos pares e, consequentemente à depressão. Assim, fatores que, como a não 
conformidade de gênero, afetam o estabelecimento de relações sociais e a inserção em grupos e podem contribuir com uma queda na qualidade de vida destas crianças.

Focando em crianças não conformes de gênero, Beijsterveldt et al. (2006) mediram o risco destas crianças apresentarem problemas emocionais e comportamentais. Estes autores encontraram que estas crianças apresentaram mais risco de problemas emocionais, como ansiedade, e comportamentais, como agressividade e quebra de regras, que as crianças com comportamento típico de gênero. Apesar de mais meninas apresentarem não conformidade de gênero, tais problemas emocionais e comportamentais pareceram mais frequentes entre os meninos, o que pode indicar uma maior tolerância ao comportamento atípico feminino do que ao masculino (o que é corroborado pelos dados de Fagot, 1977). Zucker, Wilson-Smith, Kurita e Stern (1995) utilizando histórias de crianças fictícias e a classificação de quanto cada criança participante desejaria ser amiga das personagens, identificaram que as crianças do sexo masculino tendem a preferir como amigos aqueles com maior índice de masculinidade. A presença de um único comportamento tipicamente feminino já influenciava a valorização desta criança pelo grupo, de modo que quanto mais feminino, menos era desejado como amigo. Inversamente, quanto mais masculina fosse uma criança do sexo feminino, mais ela era preferida pelos participantes do sexo masculino. Resultados inversamente similares foram obtidos com participantes do sexo feminino. Novamente, a rejeição de meninas masculinas foi muito menor entre as participantes do sexo feminino do que a rejeição de meninos femininos por participantes do sexo masculino.

Pode-se perceber, portanto, que estudos sobre a configuração das diferenças de gênero e seus impactos são de grande relevância tanto para a compreensão do desenvolvimento destes padrões quanto para a intervenção favorecendo a qualidade de vida destas crianças.

Finnegan et al. (1991) destacam a importância de haver instrumentos adequados para a medida de padrões típicos de gênero (complementares à entrevista clínica). Segundo estes autores, os instrumentos deveriam ser de fácil aplicação, mas provendo informações suficientes para contribuir com o trabalho clínico. Da mesma forma, o ideal seria que os resultados obtidos permitissem a visualização de uma distribuição contínua entre níveis de feminilidade e masculinidade, ao invés de valores dicotômicos.

Fridell et al. (2006) testaram a validade de um instrumento de fácil aplicação direta a crianças, a Entrevista Estruturada de Preferências de Parcerias e Estilos de Brincadeiras (PPPSI). Este instrumento foi desenvolvido por Alexander e Hines (1994) e utilizado por outros autores (Fridell et al., 2006; Pasterski, 2002; Zucker, 2005), apresentando elevada correlação entre o gênero (masculinidade/feminilidade) e as respostas obtidas, independentemente do sexo dos participantes. Outra medida de fácil aplicação consiste no teste "Desenho da Figura Humana" (Zucker, Finegan, Doering, \& Bradley, 1983), que já foi utilizado previamente por diversos autores (Franco, Karp, \& Schottenbauer, 2005; Fridell et al., 2006) para identificação de conformidade de gênero, apresentando elevada correlação com preferências de brincadeiras e vestuário. A literatura indica que o sexo da primeira pessoa desenhada tende a ser aquele com quem o participante mais se identifica.

Considerando que a literatura atual sobre a relação entre sexo e preferência de pares e brincadeiras é estritamente internacional, o presente estudo teve por objetivo avaliar se o PPPSI e o Desenho da Figura Humana seriam instrumentos válidos para investigação de padrões típicos de gênero também no Brasil, discutindo se os mesmos itens elaborados e aplicados em outras culturas também indicariam uma correlação entre padrões e o sexo de crianças de Belém-Pará-Brasil. Esta investigação é relevante para contribuir com a discussão da generalidade e universalidade da relação entre sexo, identidade de gênero e padrões típicos de gênero, independentemente da cultura na qual o indivíduo está inserido.

\section{Método}

\section{Participantes}

A pesquisa foi realizada com 234 crianças, entre 09 e 11 anos de idade. Destas, 120 eram do sexo feminino e 114 do sexo masculino. Todos os participantes foram selecionados a partir da listagem de alunos de salas de aula de $4^{\mathrm{a}} / 9$ e $5^{\mathrm{a}} / 9$ séries do ensino fundamental de escolas particulares, nas quais foi obtida autorização da instituição e dos pais mediante assinatura do Termo de Consentimento Livre e Esclarecido.

\section{Ambiente}

A aplicação dos instrumentos foi feita a grupos de cerca de 30 alunos nas suas salas de aula. As carteiras eram dispostas de forma que possibilitasse o sigilo das respostas.

\section{Material e Equipamentos}

Foi utilizada uma encadernação padronizada para esta pesquisa, contendo dois instrumentos. Os instrumentos utilizados foram: o teste "Desenho da Figura Humana" (adaptado) e a Entrevista Estruturada de Preferências de Parcerias e Estilos de Brincadeiras (PPPSI - sigla em inglês).

Quanto ao teste "Desenho da Figura Humana", foi aplicada apenas a primeira parte do teste, ou seja, foi solicitado o desenho de "uma pessoa". Foi entregue uma folha de papel A4 em branco (primeira página da encadernação, depois da capa) e um lápis de grafite. Esta folha continha um comando digitado no topo, onde se lia "Desenhe uma pessoa. Pode ser qualquer pessoa que você quiser!" 
A Entrevista Estruturada de Preferências de Parcerias e Estilos de Brincadeiras (PPPSI) iniciava logo em seguida. A entrevista consiste na apresentação de 40 situações de escolha, nas quais são dispostas duas figuras bidimensionais e em preto-e-branco. Na Condição 1, há 14 pares com o desenho de um menino e de uma menina, ambos com o mesmo brinquedo. Na Condição 2, há 13 pares com o desenho de crianças sexualmente ambíguas, sendo que em uma figura há um estímulo tipicamente masculino e na outra, um feminino. Destes 13 pares, quatro estímulos são brinquedos; quatro são tipos de brincadeiras; quatro são níveis de atividade; e no último par há a figura de um grupo de quatro crianças, sendo em um desenho todas do sexo masculino e no outro do sexo feminino. Na Condição 3, há 13 pares de figuras conflitantes, isto é, com o desenho de uma figura feminina junto a um estímulo masculino e o outro com uma figura masculina junto a um estímulo feminino.

\section{Procedimento}

Após aprovação do Comitê de Ética em Pesquisa em Seres Humanos do Instituto de Ciências da Saúde da Universidade Federal do Pará (Protocolo N 039/09 CEPICS/UFPA), foi obtida a autorização das escolas para a realização da pesquisa. Foi enviada, então, uma carta explicativa do projeto, junto ao Termo de Consentimento Livre e Esclarecido a todos os pais ou responsáveis de alunos das séries estudadas. Após o recebimento dos termos assinados, foi agendada uma visita à escola para aplicação dos instrumentos.

No dia programado, cada aluno recebia a encadernação, na qual era atribuído um código (identificando o sexo da criança e atribuindo um número arbitrário), possibilitando a análise anônima dos instrumentos. Após a apresentação das pesquisadoras, os participantes eram informados que iriam contribuir respondendo sobre suas preferências de brincadeiras, preenchendo o instrumento juntos, avançando de página conforme orientados.

Primeiramente, os participantes foram convidados a fazer o desenho de uma pessoa em uma folha de papel com lápis disponibilizado para eles. Eram orientados, ainda, a atribuir um nome ao desenho de modo a favorecer a identificação do sexo do mesmo. Quando a criança solicitava por desenhar mais pessoas, ela era autorizada, mas uma marcação era feita sinalizando qual havia sido o primeiro desenho realizado.

Em seguida, as crianças eram orientadas a virar a página. Era lido comando da primeira situação de escolha do PPPSI (por exemplo, "Tip gosta de brincar de luta. Top gosta de jogar jogos de tabuleiro. Com quem você prefere brincar, Tip ou Top?") e as crianças marcavam a resposta no instrumento. Após o encerramento, todas as crianças devolviam os instrumentos para as pesquisadoras e retornavam à aula normalmente.

\section{Análise de Dados}

Foram realizados testes estatísticos descritivos e de significância (foi escolhido o qui quadrado, em função da natureza dos dados, sendo aceito como significativos valores inferiores a 0,05 ).

Foi calculada a média das respostas obtidas nos pares de cada condição prevista pelo instrumento. A média obtida por cada participante nas condições foi tratada, então, como índices para a Condição 1, Condição 2 e Condição 3 , com amplitude entre 1 e 2 (sendo 1 a pontuação mais feminina e 2 a mais masculina, conforme o que era esperado pela estrutura do instrumento). A média dos resultados obtidos nas três condições foi considerada como o Índice de Feminilidade-Masculinidade de cada participante (IFM). Assim, foi possível analisar o resultado de cada participante a partir do seu nível de feminilidade/ masculinidade em cada condição e no geral (IFM).

\section{Resultados e Discussão}

Com relação aos desenhos feitos pelas crianças, $82,5 \%$ das meninas desenharam primeiro uma pessoa do sexo feminino, $13,3 \%$ do sexo masculino e em $4,2 \%$ dos desenhos não foi possível identificar o sexo da figura (personagens andróginos de desenhos animados, por exemplo). Entre os meninos, $76,3 \%$ desenharam uma pessoa do sexo masculino e $21,1 \%$ do sexo feminino (com 2,6\% das respostas sem ser identificado o sexo do desenho). Desta forma, observou-se que o sexo do participante e o sexo do primeiro desenho feito estão significativamente relacionados $\left(\chi^{2} 1=94,632, p<0,001, \mathrm{~V}=0,647\right)$.

Quanto ao PPPSI, tem sido encontrada uma forte correlação entre o gênero do participante e o parceiro, bem como entre o gênero e o estímulo escolhidos. Na condição conflitante, os resultados obtidos na literatura até o momento indicam que há uma predominância por escolhas baseadas no estímulo, em detrimento de quem é o parceiro (Fridell et al., 2006; Pasterski, 2002; Zucker, 2005). O presente estudo encontrou resultados similares, que se encontram descritos em seguida.

Quanto à Condição 1, na qual ambas as opções apresentavam a mesma brincadeira, mas com bonecos de sexos opostos, participantes do sexo masculino tenderam a optar por figuras que continham bonecos do sexo masculino, enquanto as participantes do sexo feminino tenderam a optar por figuras que continham bonecos do sexo feminino (Figura 1). Este resultado foi obtido tanto com o índice individual da Condição1 $\left(\chi^{237}=192,664\right.$, $p<0,001, \mathrm{~V}=0,909)$ quanto com cada uma das 14 situações de escolha que compõem esta Condição. Obteve-se como resultado uma distribuição bimodal, onde 43 das 120 meninas escolheram figuras do sexo feminino em todas as situações e 41 dos 114 meninos escolheram figuras do sexo masculino em todas as situações. 


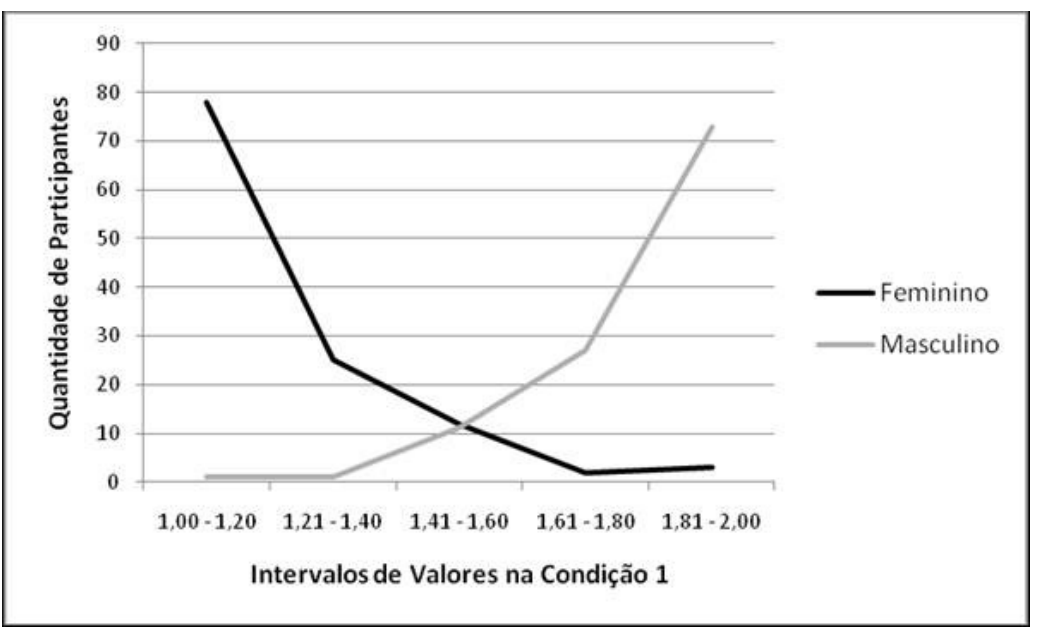

Figura 1. Quantidade de indivíduos em cada intervalo de valores da condição 1 (variando de 1,00 - mais feminino a 2,00 - mais masculino), de acordo com o sexo dos participantes.

Da mesma forma, o sexo do desenho feito pela criança estava significativamente correlacionado ao sexo do boneco escolhido pela criança nas figuras da Condição 1 $\left(\mathrm{x}^{2} 37=102,935, p<0,001, \mathrm{~V}=0,675\right)$.

$\mathrm{Na}$ Condição 2, na qual a figura não permitia a identificação do sexo do par da brincadeira mas contrapunha como opções uma brincadeira tipicamente masculina e outra tipicamente feminina, também foi obtida correlação entre os escores das escolhas e o sexo do participante $\left(\mathrm{x}^{2} 35=178,166, p<0,001, \mathrm{~V}=0,873\right)$ e entre os escores das escolhas e o sexo do desenho feito pelo participante $\left(x^{2} 35=100,812, p<0,001, \mathrm{~V}=0,668\right)$. Apesar da relação ter sido significativa também na Condição 2 , pode-se perceber que houve maior variação entre as respostas dos participantes, relativamente à Condição 1 (Figura 2).

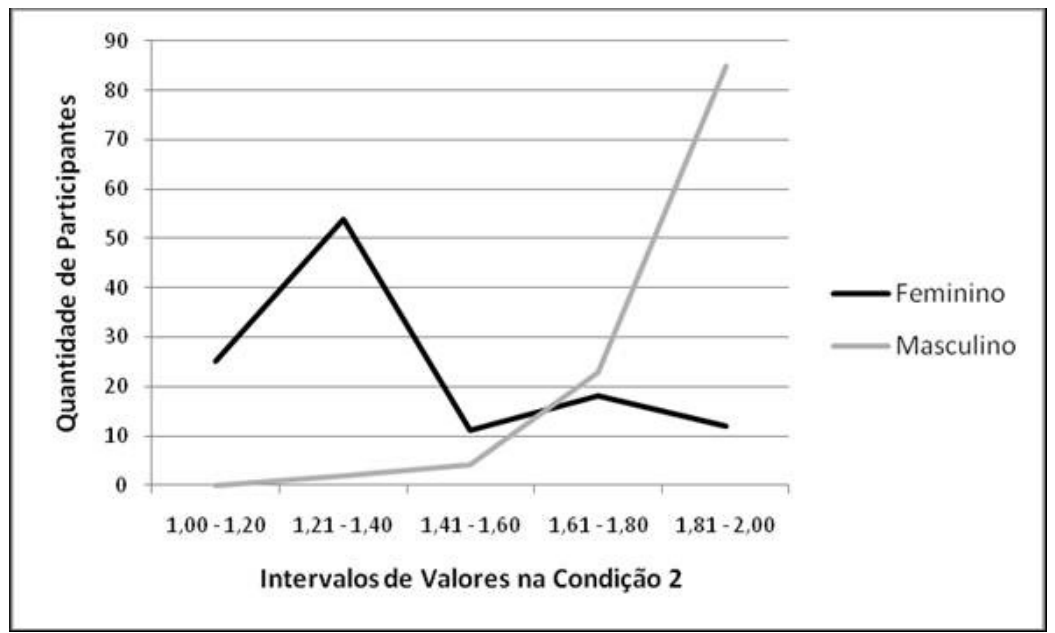

Figura 2. Quantidade de indivíduos em cada intervalo de valores da condição 2 (variando de 1,00 - mais feminino a 2,00 - mais masculino), de acordo com o sexo dos participantes.

Analisando cada uma das 13 situações de escolha, não foi obtida correlação em apenas uma das situações, a que contrapunha "Andar calmamente pelo quintal e explorar o espaço" a "Correr pelo quintal" $\left(\mathrm{x}^{2} 1=2,533, p=0,111\right.$, $\mathrm{V}=104)$. Nesta situação, $52,5 \%$ das respostas das meninas foram "Andar calmamente pelo quintal e explorar o espaço" (resposta tida como tipicamente feminina - TF) e 47,5\% escolheram "Correr pelo quintal" (resposta tida como tipicamente masculina - TM). Ainda, nesta situação, 57,9\% dos meninos realizaram a escolha tipicamente masculina, enquanto $42,1 \%$ destes optaram pela atividade tipicamente feminina. Pode-se questionar que a expressão "explorar o espaço" pode ter tido múltiplas interpretações de que atividades estariam envolvidas, de modo a gerar padrões tão diversos de respostas, tanto entre meninos quanto entre meninas. 
$\mathrm{Na}$ Condição 3, na qual uma atividade tipicamente masculina desempenhada por uma figura feminina era contraposta a uma atividade tipicamente feminina desempenhada por uma figura masculina, também foi obtida relação entre os escores das escolhas e o sexo do participante $\left(\mathrm{x}^{2} 33=145,284, p<0,001, \mathrm{~V}=0,788\right)$ e entre os escores das escolhas e o sexo do desenho feito pelo participante $\left(x^{2} 33=82,763, p<0,001, V=0,605\right.$; Figura 3$)$.
Para esta condição, foi utilizado para o cálculo da relação entre o sexo relativo à atividade e não ao par presente na figura (conforme descrito na literatura, por exemplo Fridell et al., 2006; Pasterski, 2002; Zucker, 2005). Assim, pode-se entender que os participantes tenderam a responder de acordo com as atividades apresentadas e não com os parceiros presentes nas respectivas atividades.

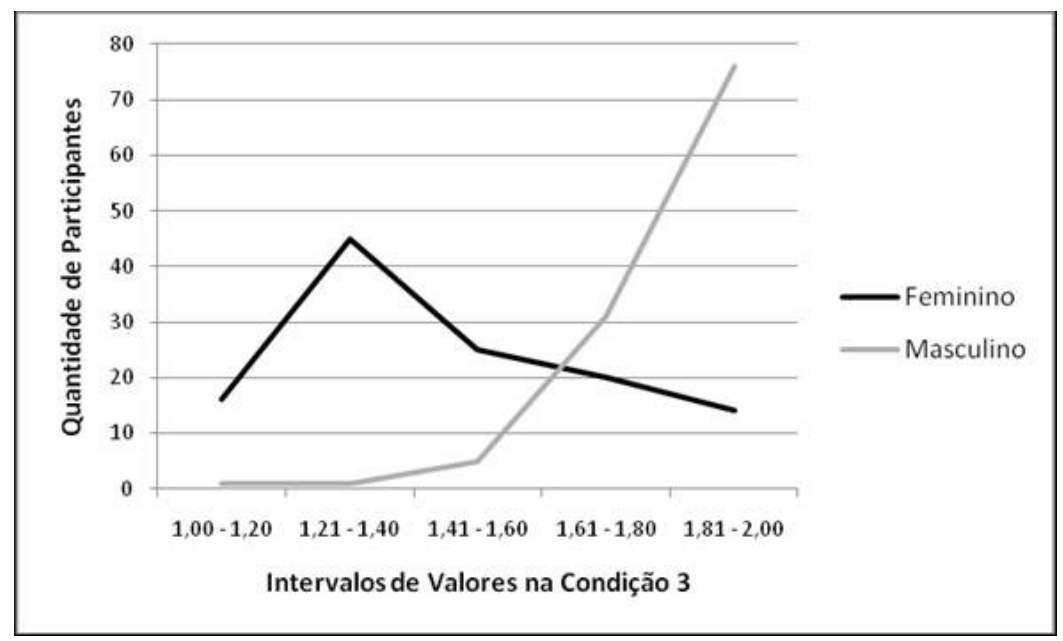

Figura 3. Quantidade de indivíduos em cada intervalo de valores da condição 3 (variando de 1,00 - mais feminino a 2,00 - mais masculino), de acordo com o sexo dos participantes.

A preferência pela atividade e não pelo sexo do parceiro corrobora a hipótese evolucionista, segundo a qual a formação de grupos seria uma consequência de padrões comportamentais comuns e não da formação de uma identidade sexual prévia. Este dado é coerente, assim, com estudos anteriores, demonstrando que crianças preferem brincar com outras crianças que adotam estilos de brincadeira similares aos seus, independentemente do sexo (Fridell et al., 2006; Pasterski, 2002; Zucker, 2005).

Contudo, analisando individualmente cada uma das 13 situações de escolha, não foi obtida relação em duas delas. Quando contraposto "Jogos de correr e pegar" (TM) e "Resolver quebra-cabeças" (TF) não houve relação com o sexo do participante $\left(\mathrm{x}^{2} 1=1,202, p=0,273, \mathrm{~V}=0,72\right)$. Apenas $23,3 \%$ das meninas escolheram a opção TF, enquanto as demais $76,7 \%$ optaram pela atividade TM. De forma similar, $82,5 \%$ dos meninos optaram pela atividade TM, tendo apenas $17,5 \%$ das escolhas sido TF. Percebeu-se então que crianças de ambos os sexos tenderam a preferir "Jogos de correr e pegar" a "Resolver quebracabeças". Isto pode ser resultado de um padrão cultural, podendo ser um indício de que quebra-cabeças não sejam brinquedos muito comuns para as crianças brasileiras, independentemente do sexo. Para confirmar esta hipótese, outras investigações teriam que ser feitas. Caso a hipótese esteja correta, seria pertinente adaptar este item do instrumento, substituindo a atividade "Resolver que- bra-cabeças" por outra atividade similar, mas mais presente no contexto brasileiro, para identificar se o resultado aqui encontrado representa a recusa em engajar-se nesta atividade específica ou a preferência por atividades mais turbulentas (no caso, "Jogos de correr e pegar") inclusive por crianças do sexo feminino. Esta adaptação serviria de teste para identificar que elementos afetaram a escolha das participantes.

Quando contraposto "Andar pelo quintal e explorar o espaço" (TF) e "Correr pelo quintal" (TM) também não foi obtida relação com o sexo do participante $\left(\mathrm{x}^{2} 3=2,5888\right.$, $p=0,46, \mathrm{~V}=0,105)$, de modo que $46,7 \%$ das meninas optaram pela atividade TF e $51,7 \%$ pela TM, enquanto que $42,1 \%$ dos meninos optaram pela atividade TF e $57,9 \%$ pela TM. Neste caso, percebeu-se que crianças de ambos os sexos não apresentaram preferência clara entre as atividades "Andar pelo quintal e explorar o espaço" e "Correr pelo quintal". Novamente, percebeu-se a ausência de relação quando estes dois itens são contrapostos. Como isto ocorreu tanto na Condição 2 quanto na Condição 3, pode-se interpretar que isto indica variabilidade na preferência das atividades, independentemente da explicitação do sexo do par.

Conforme esperado, tendo por base as relações obtidas independentemente das condições, o sexo do participante e o sexo do desenho feito também estavam relacionados com o Índice de Feminilidade e Masculinida- 
de - IFM $\left(\mathrm{x}^{2} 167=230,998, p=0,001, \mathrm{~V}=0,996\right.$; ver Figura 4). O IFM obtido entre as participantes do sexo feminino foi em média 1,33 (Desvio Padrão 0,195), com moda de 1,2. Entre os participantes do sexo masculino, a média do IFM foi igual a 1,84 (Desvio Padrão 0,115), com moda de 1,9 .

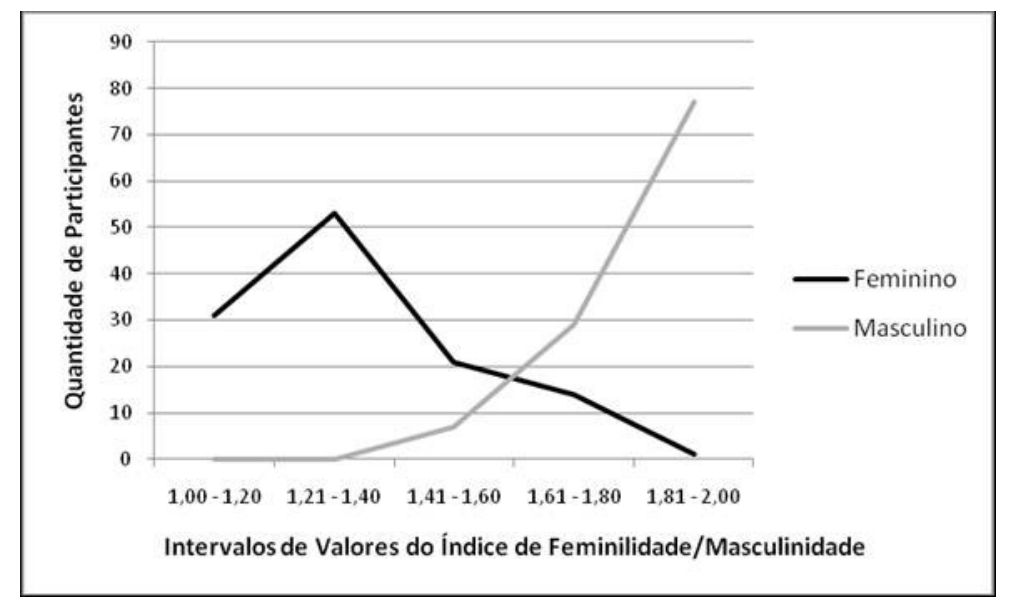

Figura 4. Quantidade de indivíduos em cada intervalo de valores do índice de feminilidade/masculinidade (variando de 1,00 - mais feminino a 2,00mais masculino), de acordo com o sexo dos participantes.

Vale ressaltar que $21 \%$ das participantes do sexo feminino apresentaram IFM superior a 1,5 - isto é, mais próximo da extremidade masculina que da feminina. Por sua vez, apenas $2 \%$ dos participantes do sexo masculino apresen-taram IFM inferior a 1,5. Este dado é compatível com os dados da literatura de que a frequência de indivíduos não conformes de gênero é baixa em geral, sendo mais comum crianças do sexo feminino engajadas em atividades tipicamente masculinas do que o inverso. Não foram investigados, no presente estudo, os impactos sociais destes padrões.

\section{Considerações Finais}

Os dados obtidos por Alexander e Hines (1994), Fridell et al. (2006), Pasterski (2002) e Zucker (2005) foram similares aqueles encontrados no presente estudo, indicando que as diferenças culturais entre o país em que o instrumento foi elaborado (Estados Unidos da América), países nos quais já foi aplicado (Canadá e Inglaterra) e, agora, o Brasil, não foram relevantes nos dados obtidos a partir da presente amostra, de modo a não interferir nas relações obtidas (com exceção do brinquedo "quebra-cabeças"). Contudo, faz-se necessário a replicação do estudo com objetivo de validação nacional do instrumento.

Esta coerência entre os resultados obtidos em culturas distintas é essencial para fundamentação da perspectiva evolucionista. Como discute Alexander (2003), se há padrões sexualmente dimórficos que foram selecionados a partir de sua função evolutiva no AAE, estes deveriam ser observados, mesmo que com algumas variações, universalmente entre os indivíduos da espécie, independentemente de variações culturais. Assim, este estudo é mais um passo nesta direção. Sugere-se, desta forma, que sejam realizados novos estudos similares, em culturas distintas, comparando os resultados obtidos.

Uma limitação deste estudo se refere ao fato que o instrumento foi aplicado de forma coletiva. Apesar de terem sido tomadas precauções para garantir que cada criança visualizasse apenas o seu instrumento e que tivesse certeza do sigilo e anonimato das informações, é possível que algumas crianças não tenham respondido com padrões não conformes de gênero por receio de implicações sociais (o que é compatível com os resultados obtidos por Zucker et al., 1995).

Os instrumentos utilizados na presente pesquisa demonstraram-se de fácil aplicação e análise, possuindo relações significativas com o sexo do participante. Conforme afirmam Finnegan et al. (1991), esta caracterização é fundamental para que estes instrumentos possam ser utilizados no trabalho clínico. Assim, pesquisas posteriores, com um viés clínico, podem envolver a aplicação individual do PPPSI e, juntamente a este, a avaliação de outros instrumentos voltados a aspectos relativos à qualidade de vida, autoestima, identidade e socialização da criança. Esta derivação clínica do presente estudo pode se demonstrar um importante suporte para a atuação de profissionais frente ao ostracismo social enfrentado por crianças não conformes de gênero (conforme relatado por Beijsterveldt et al., 2006; Cohen-Kettenis et al., 2003; Van Beek et al., 2006; Zucker et al., 1995).

Este trabalho contribui, ainda, ao indicar que há uma preferência por estilos de brincar dependente do sexo da criança e que esta preferência antecede a formação grupal (como indicado por Liss, 1981); consequentemente o segregacionismo sexual decorreria do agrupamento por afinidade e não o inverso (Menezes et al., 2010). 
Compreender aspectos evolutivos e biológicos que precedem o dimorfismo sexual comportamental é fundamental para que os dados obtidos neste tipo de investigação possam ser plenamente discutidos. A escolha por atividades mais turbulentas por meninos e por atividades domésticas por meninas pode não ser uma simples imposição cultural, mas remontar à toda estrutura orgânica dos sexos (especialmente o funcionamento hormonal) e à função de sobrevivência da espécie, quando no AAE (Alexander, 2003). Da mesma forma, não se pode defender que estes padrões estejam pré-definidos de forma estanque, pois a suscetibilidade à influência ontogenética e cultural é fundamental para a adaptação constante de cada organismo ao seu ambiente presente (Shirley \& Campbell, 2000).

\section{Referências}

Alexander, G. M. (2003). An evolutionary perspective of sextyped toy preferences: Pink, blue, and the brain. Archives of Sexual Behavior, 32(1), 7-14.

Alexander, G. M., \& Hines, M. (1994). Gender labels and play styles: Their relative contribution to children's selection of playmates. Child Development, 85, 869-879.

Alexander, G. M., Wilcox, T., \& Woods, R. (2009). Sex differences in infants' visual interest in toys. Archives of Sexual Behavior, 38, 427-433.

American Psychiatric Association. (1995). Manual diagnóstico e estatístico de transtornos mentais - DSM IV (4. ed.). Porto Alegre, RS: Artes Médicas.

Bailey, J. M., Bechtold, K. T., \& Berenbaum, S. A. (2002). Who are tomboys and why should we study them? Archives of Sexual Behavior, 31, 333-341.

Beijsterveldt, C. E. M. van, Hudziak, J. J., \& Boomsma, D. I. (2006). Genetic and developmental influences on cross gender behaviors. A study of Dutch twins at ages 7 and 10. Archives of Sexual behavior, 35, 647-658.

Bianchi, B. D., \& Bakeman, R. (1978). Sex-typed affiliation preferences observed in preschoolers: Traditional and open school differences. Child Development, 49, 910-912.

Burghardt, G. M. (2004). The genesis of animal play: Testing the limits. Cambridge, MA: Massachusetts Institute of Technology Press.

Bussey, K., \& Bandura, A. (1999). Social cognitive theory of gender development and differentiation. Psychological Review, 106, 676-713.

Cohen-Kettenis, P. T., Owen, A., Kaijseer, V. G., Bradley, S. J., \& Zucker, K. J. (2003). Demographic characteristics, social competence, and behavior problems in children with gender identity disorder: A cross-national, cross-clinic comparative analysis. Journal of Abnormal Child Psychology, 31(1), 41-53.

Conti, L., \& Sperb, T. M. (2001). O brinquedo de pré-escolares: Um espaço de ressignificação cultural. Psicologia: Teoria e Pesquisa, 17(1), 59-67.

Fagot, B. I. (1977). Consequences of moderate cross-gender behavior in preschool children. Child Development, 48, 902 907.

Finnegan, J. A. K., Niccols, G. A., Zacher, J. E., \& Hood, J. E. (1991). The Play Activity Questionnaire: A parent report measure of children's play preference. Archives of Sexual Behavior, 20(4), 393-408.
Franco, F., Karp, S. A., \& Schottenbauer, M. A. (2005). The Spanish version of the Draw-A-Person Questionnaire (DAPQ). Hispanic Journal of Behavioral Sciences, 27(4), 455-470.

Fridell, S. R., Owen-Anderson, A., Johnson, L. L., Bradley, S. J., \& Zucker, K. J. (2006). The playmate and play style preferences structured interview: A comparison of children with gender identity disorder and controls. Psychological Review, 107(3), 411-429.

Golombok, S., Rust, J., Zervoulis, K., Croudace, T., Golding, J., \& Hines, M. (2008). Developmental trajectories of sextyped behavior in boys and girls: A longitudinal general population study of children aged $2.5-8$ years. Child Development, 79(5), 1583-1593.

Iervolino, A. C., Hines, M., Golombok, S. E., Rust, J., \& Plomin, R. (2005). Genetic and environmental influences on sex-typed behavior during the preschool years. Child Development, 76, 826-840.

Jacklin, C. N., DiPietro, J. A., \& Maccoby, E. E. (1984). Sextyping behavior and sextyping pressure in child/parent interaction. Archives of Sexual Behavior, 13(5), 413-425.

Jadva, V., Hines, M., \& Golombok, S. (2010). infants' preferences for toys, colors, and shapes: Sex differences and similarities. Archives of Sexual Behavior, 39, 1261-1273.

Liss, M. B. (1981). Patterns of toy play: An analysis of sex Differences. Sex Roles, 7(11), 1143-1150.

Menezes, A. B. C., Brito, R., Figueira, R. A., Bentes, T. F., Monteiro, E. F., \& Santos, M. C. (2010). Compreendendo as diferenças de gênero a partir de interações livres no contexto escolar. Estudos de Psicologia (Natal), 15, 79-87

Pasterski, V. L. (2002). Development of gender role behaviour in children: Prenatal hormones and parental socialization. (Unpublished doctoral disertation). City University, London.

Pomerleau, A., Bolduc, D., Malcuit, G., \& Cossette, L. (1990). Pink or blue: Environmental gender stereotypes in the first two years of life. Sex Roles, 22(5-6), 359-367.

Shirley, L. J., \& Campbell, A. (2000). Same-sex preference in infancy: Visual preference for sex-congruent stimuli at three months. Psychology, Evolution \& Gender, 2(1), 3-18.

Van Beek, Y., van Dolderen, M. S. M., \& Dubas, J. J. S. D. (2006). Gender-specific development of nonverbal behaviours and mild depression in adolescents. Journal of Child Psychology and Psychiatry, 47(12), 1271-1283.

Van de Beek, C., van Goozen, S. H. M., Buitelaar, J. K., \& Cohen-Kettenis, P. T. (2009). Prenatal sex hormones (maternal and amniotic fluid) and gender related play behavior in 13-month-old infants. Archives of Sexual Behavior, 38, 6-15.

Zucker, K. J. (2005). Measurement of psychosexual differentiation. Archives of Sexual Behavior, 34, 375-388.

Zucker, K. J., Finegan, J. A. K., Doering, R. W., \& Bradley, S. J. (1983). Human figure drawings of gender-problem children: A comparison to siblings, psychiatric and normal controls. Journal of Abnormal Child Psychology, 11(2), 287-298.

Zucker, K. J., Wilson-Smith, D. N., Kurita, J. A., \& Stern, A. (1995). Children's appraisals of sex-typed behavior in their peers. Sex Roles, 33(11-12), 703-725. 